\title{
HOW TO EXTRACT USEFUL INFORMATION ABOUT THE DECAY OF BASS RELIEVES IN ARCHAEOLOGICAL AREA
}

\author{
E. S. Malinverni ${ }^{1}$, R. Pierdicca ${ }^{1}$, F. Di Stefano ${ }^{1}$, M. Sturari ${ }^{2}$, M. Mameli ${ }^{2}$, E. Frontoni ${ }^{2}$, R. Orazi ${ }^{3}$, F. Colosi ${ }^{3}$ \\ ${ }^{1}$ Department of Construction, Civil Engineering and Architecture, Università Politecnica delle Marche, Ancona, Italy \\ (e.s.malinverni, r.pierdicca)@univpm.it, f.distefano@pm.univpm.it \\ ${ }^{2}$ Department of Information Engineering, Università Politecnica delle Marche, Ancona, Italy \\ m.sturari@pm.univpm.it, e.frontoni@univpm.it, mameli.1.marco@gmail.com \\ ${ }^{3}$ Institute for technologies applied to cultural heritage, Consiglio Nazionale delle Ricerche, Rome, Italy \\ (francesca.colosi, roberto.orazi)@itabc.cnr.it
}

\begin{abstract}
KEY WORDS: Change Detection, Color Decay, 3D Reconstruction, Archaeology, Photogrammetric application, k-means clustering
\end{abstract}

\begin{abstract}
:
Cultural Heritage goods represent the memory and the history of the civilization. Notwithstanding, there are not sufficient public resources to guarantee their preservation and maintenance. Nowadays between several geomatic techniques available, the pillar for the preservation of mankinds heritage is the low cost close photogrammetric acquisition. The advantages of virtual reconstructions based on Multi View Stereo (MVS) and Structure from Motion (SfM) algorithms is extended from the heritage documentation to its virtualization or modelling. The digital preservation of archaeological sites is committed in more agile and friendly procedures that give automatic extraction of information to perform in depth analysis over ancient artefacts. In the field of $\mathrm{CH}$ research, the characterization and classification of the conservation state of the materials composing the surface of the artefacts are essential to study their damage. The first step for conservation state of a goods is the study of the changes in different times. The possibility to automatically study this time modification due to different factor represents a key point for the archaeologists' work. With this in mind, the aim of this work is to propose a completely automatic methods for change detection between three data set acquired in different époques. The work flow applied is based on the unsupervised clustering techniques applied on a combination of two type of differences images. The results, unlike the objective, demonstrate that the unsupervised methods are not effectiveness in the $\mathrm{CH}$ study, instead of the supervised methods that outperforms in terms of reliability of results.
\end{abstract}

\section{INTRODUCTION}

Archaeology is the most important cultural heritage for most Latin American countries. The defense, the conservation and the re- search of many important archaeological findings is fundamental to identify and understand the different cultures that have existed throughout history and that have disappeared today. Unfortunately for many environmental and local problems it is very difficult to carry out a good conservation and restoration work, registering and cataloguing archaeological goods. Notwithstanding, there are not sufficient public resources to guarantee their preservation and maintenance. The documentation of a Cultural Heritage $(\mathrm{CH})$ is defined as the action of acquiring, processing, presenting and recording the necessary data for the determination of position, shape and size of a monument in the three-dimensional space at a particular temporal moment. Nowadays between several geomatic techniques available, the pillar for the preservation of mankinds heritage is the low cost close photogrammetric acquisition. The advantages of virtual reconstructions based on Multi View Stereo (MVS) and Structure from Motion (SfM) algorithms is extended from the heritage documentation to its virtualization or modelling. In recent years, this approach has become dominant when dealing with archaeological sites in precarious condition and often inaccessible. Their digital preservation is committed in more agile and friendly procedures that give automatic extraction of information to perform in depth analysis over ancient artefacts. In the field of $\mathrm{CH}$ research, the characterization and classification of the conservation state of the materials composing the surface of the artefacts are essential to study their damage. Besides providing a unique opportunity to produce reality based digital replicas, photogrammetry even allows to create ortho-images with very high quality. Their resolution can be exploited to perform image analysis that might support archaeologist in their work of understanding material decay; in fact, sometimes a naked eye approach may result insufficient and the support of image classification techniques may result fundamental. In this work, the methodology proposed in (Malinverni et al., 2017) for the evaluation of color degradation between the decorative representations acquired at different époques is extended. A new dataset has been collected in 2017, on the same bas-relieves located at Huaca de la Luna in Peru, to make a comparison with the ones collected in 2006 and 2012. This is done to evaluate if the indications of changes obtained in 2012 are confirmed in 2017, highlighting an evolution of decay or false positives of the methodology. The previous method has been, moreover, compared with an unsupervised approach, used as a benchmark. Results demonstrate that the unsupervised methods are not effectiveness in the $\mathrm{CH}$ study, instead of the supervised methods that outperforms in terms of reliability and accuracy. The paper presents the following structure, in the Sec 2. report a description of the state of the art in the change detection application from $\mathrm{CH}$ point of view and from the computer

\footnotetext{
* Corresponding author
} 
vision point of view; Sec 3. explain the application context; Sec 4. describes the work flow applied and the process useful to the change detection from a computer vision point of view. At the end the Sec 5. show the results obtained and conclude with the effectiveness of the previous work (Malinverni et al., 2017) respect to a completely automatic work flow.

\section{RELATED WORK}

The main purpose of this work is to perform and compare change detection procedures able to provide information about the changes of a surface in different époques of acquisition. The topic of analysing the dynamics of changing is not recent itself. The research carried by Burroni et al. (Burroni et al., 2002) was based on the direct ex- amination of a representative sample of stone tool by-products, and on the identification of all surface alteration features. The main issue was concerned with the automation of the diagnosis process. Thanks to the large adoption of geomatics techniques for indepth analysis, the work of Appolonia et. al (Appolonia et al., 2006) is developed on image processing tools used to improve and make semiautomatic the study of chemical decay causing visible changes in colour of some regions. As a demonstration of this, in (Atkinson et al., 2010) geomatics techniques were broadly adopted to extract information like magnitudes (linear dimensions and surface areas) of structural decay pathology. In order to compare snapshots of the same view taken at different times, some algorithms are implemented. In (Pierdicca et al., 2018), the authors used a Deep Learning architectures for detection PV cells damaged using a new dataset of thermal images collected by drone equipped with a thermal infrared sensor. A similar architecture based on CNNs for semantic segmentation using top-view depth visual data is applied in a completely different field, to detect and track people in crowded environment (i.e. retail, airport, station, etc.) (Liciotti et al., 2018). Another work (Paolanti et al., 2018) that extract anthropometric features for the recognition of people using a top-view camera, reducing the problem of occlusions, propose the combination of multiple k-nearest neighbor classifiers based on different distance functions and feature subsets derived from depth and color images. Advanced techniques for the radiometric and geometric analysis have been experienced in (Barazzetti et al., 2010) using multispectral images and the 3D surveying of a painting. Integrating not invasive surveying techniques and evaluation methods for $\mathrm{CH}$ objects represents an innovative methodology to obtain, for example, thematic maps with the size and position of damages, how demonstrated by the research works mentioned before. The literature also provides some experiences related to archaeological field of study. In the method proposed by (Levin et al., 2010), the detection of changes is based on outlier analysis which, according to the authors, overcomes the limitations of a point-to-point differencing or of segmentation and classification of the data. In some other cases, the aid of TLS was adopted to understand the changes over time of rocks (Alba et al., 2010). It is worth also to cite the work of Cerra et. al. (Cerra et al., 2016), since it points out the necessity of using change detection techniques in this field where WorldView2 images have been used to detect the changes occurred during the civil war in Syria. According to (Lu et al., 2004), good change detection research should provide the following information: i) area change and change rate; ii) spatial distribution of changed types; iii) change trajectories of land-cover types; and iv) accuracy assessment of change detection results.

\section{THE CASE STUDY}

The Huaca de la Luna (Huaca of the Moon) is located along the north coast of Peru, near the city of Trujillo. It is the most famous and the best preserved stepped pyramid built by the Moche population, that occupied the coastal zone between the first and the eighth century A.D (Bourget et al., 2009, Uceda et al., 2010).

The huacas, built with thousands of mud bricks (adobe), had a religious and administrative function. These monuments were renewed at each change of power and according to a ritual calendar cycle. The existing huaca was completely filled up and covered by a high layer of adobe in order to erect a new pyramid. Higher and wider than previous one. The Huaca de la

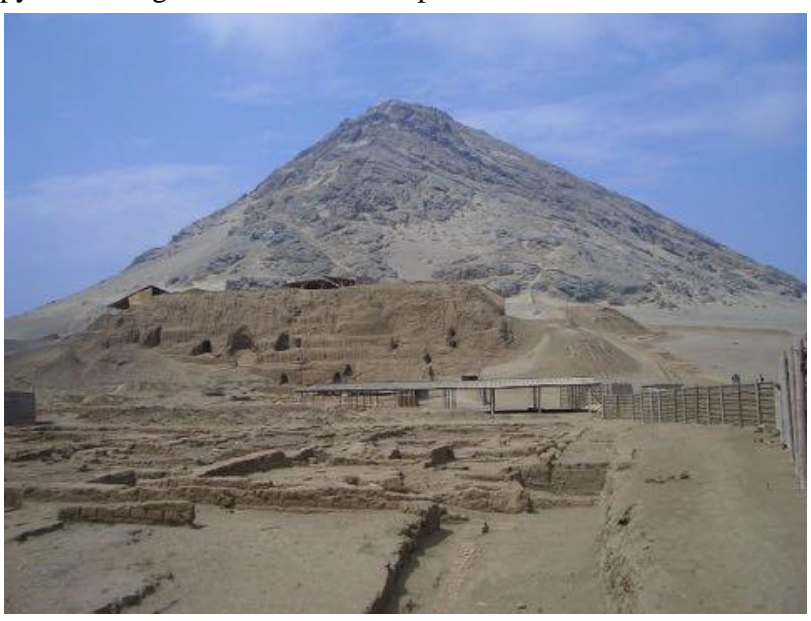

Figure 1. Huaca de la Luna

Luna is composed by two different temples which clearly mark the existence of two periods of Moche occupation. The first one, the most important sacred building in Moche society, was organized in plazas, covered patios and enclosures linked by corridors and ramps with murals or friezes painted in striking colors. The old temple underwent five construction phases, spanning almost 600 years (50-600 AD) (Uceda 2001, Uceda et al., 2016). After the Spanish conquest in the sixteenth century these ceremonial sites were abandoned and for the next four centuries, lay exposed to the elements, including the El Nio phenomenon. The adobe structures deteriorated and the uppermost platforms and superficial elements were lost. Since 1991 the Huaca de la Luna has been the subject of archaeological research and conservation interventions in the frame of the Proyecto Arqueológico Huacas del Sol y la Luna (The Huacas del Sol y de la Luna Archaeological Project HSHLAP), directed by Ricardo Morales Gamarra and Santiago Useda Castillo. The HSHLAP's core are three consistent goals: a) Multidisciplinary Archaeological Research; b) Conservation of the Monument and Archaeological Finds; and c) Community Development and Outreach Programs. The primary objective of the HSHLAP is to understand the roles and characteristics of ritual and religious practices in the origins and development of the first state society in South America through the study of the Huacas de Moche religious center. The project, funded by the Backus Cultural Heritage Foundation, the Fondo de Contravalor Per-Francia, the World Monument Fund and other local partners, led to the discovery of beautiful bas-reliefs that decorated the facade and the squares of the Huaca in its various phases. The bas-reliefs, protected over centuries by the adobe walls that had sealed them, almost entirely preserve the black, yellow, red, blue and white colors (Morales et al., 1996). The 


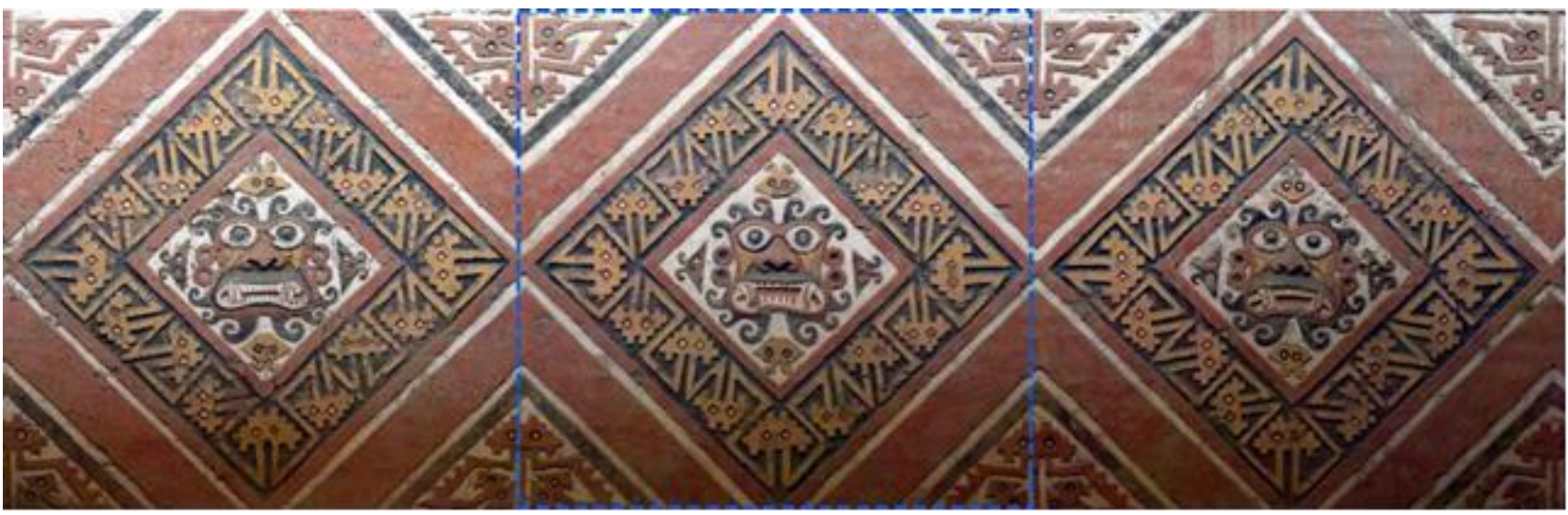

Figure 2. The image represents the orto-rectified image of the bas-relieve using the set of images of 2017. The dashed blue line depicts the portion of the entire surface that has been used for the experiments.

north facade of de Huaca de la Luna that we see today is part of the last construction done on the old temple; it bears polychrome friezes depicting deities of land and sea, as well as personages representing the functions performed in the temple. In the northeast corner of the north facade there is a corner enclosure showing murals with scenes that are excessively ornate and in apparent chaos. This is the most complex, most sophisticated, and best-conserved Moche mural art known to date (Gamarra 2003, Uceda et al., 2016). The pictorial technique was based on the use of an adhesive substance extracted from the San Pedro cactus which allowed to fix the pigments on the wall. The black color is charcoal, while all the others of mineral, inorganic nature (iron oxide, aluminum, silica and talc) (Wright 2004, Wright 2010).

\subsection{Conservation project}

The conservation of structures has evolved from arts and crafts to a scientific knowledge, broad and specialized, so that treatments and techniques can only be understood as integrated actions that require multidisciplinary teams. The safeguarding and conservation of the architectural heritage requires, today, a specialized research that must be based on modern analytical and resolving techniques. It is then that, prior to any conservative intervention on the structures, a visual analysis of the elements is carried out, diagnosing anomalies originating both from the construction materials and those acquired by abandonment of the site and exposure to the environment. The high-relieve friezes, with the related hemes around the myths and ritual practices of the Moche, were undergoing excavation, and their conservation be- came a paramount issue. According to their nature, the colors of the Huaca de la Luna are very delicate and especially sensitive to the strong wind that constantly blows from the Pacific Ocean, to the sun and to the rain that periodically can affect the coastal area. Chemicals intervention to strengthen the clay and fix the pigments are applied, until a remarkable resistance to rain, sunlight and wind is achieved. In this way the clayey earth of adobes, the floors, the monochrome plastering, the reliefs and mural paintings have acquired more physical-mechanical strength. Moreover, the monument conservation project involves a constant monitoring of the paintings, measuring the intensity of the color through a colorimeter, the surface temperature with a thermohydrometer and the wind speed and wind erosion with an anemometer (Uceda et al., 2016). According to measurements collected in the field, it seems that the bas-reliefs are not suffering loss of color, the perception that we have had on the spot in different years $(2006,2012,2017)$ is that of a minor saturation and brightness. Taking as reference the vocabulary of decay edited by ICOMOS for the monuments in stone (Vergès-Belmin, 2008), it can be said that the adobe walls of the Huaca de la Luna more exposed to the wind and light (north facade) are subject to a form of degradation due to the erosion and the discoloration of surfaces and to a phenomenon of bleaching. Thanks to the reeds covering that protects the monument and the constant work of conservation and monitoring carried out by the project Huaca della Luna, the greatest risks due to the proximity of the sea (salt crust and moist areas) are avoided. The conservation of adobe architecture requires innovative techniques and multidisciplinary efforts: archaeologists, conservators, chemists, structural engineers, imaging experts, specialists in pigment, metals, ceramics and so no. Nowadays the diagnosis of the state of conservation and pathologies of the structures is carried out by means of reports, using plans and photographs, noting in each of them locations, measures and type of architectural element, previous interventions, biological aggression and structural problem. Now to support the work of the restores on large archeological site and monitor the state of conservation of s structures, even in detail, it would be useful to introduce methods for the image acquisition and analysis of the changes, by means of not invasive automatic technologies. In this context, we believe that the possibility of using image and photogrammetric processing techniques to quantitatively evaluate the color degradation of the ancient paintings represents a further, quick and inexpensive help for a documented conservation and restoration project. For this reason change detection is one of the most important open topics for multi-temporal remote sensing technology to observe the earth. In the last year the research proposes many methods to detect the land-cover change detection. In particular this research area proceeds in two parallel way, the first one based on unsupervised clustering application and the second one use the supervised classification. For example in (Yuan et al., 2015) a new distance metric learning framework is proposed for semisupervised change detection in noisy condition. The authors demonstrate that the distance metric learning is valid method for detecting the change information from (by) high spectral features and it is robust to noise. Yuan et al use a large unbalanced data set to demonstrate the effectively of their algorithm.

In (Shao et al., 2016) a new unsupervised change detection method for multi- temporal remote sensed images is showed. This approach is based on a Robust Semi-Supervised fuzzy Cmeans (RSFCM). RSFCM introduce a pseudo-labels information instead of the information based only on intensity 
difference and spatial context. Shao et al. demonstrate the effectiveness of their algorithm with six experimental result on six different data set.

In (Mahulkar et al., 2016) the used unsupervised method needs co-registration and radiometrically corrected temporal images in input. For this absolute valued image and log ratio image is calculated and after these two images are fused with discrete wavelet transform then a normalization is done and a clusterization is applied with two cluster.

\section{MATERIALS AND METHODS}

This section is aimed at explaining the complete procedure adopted for our experiment. In deep, it starts from the description of the data-set collected to obtain the final orthoimages of bas-relieves. Afterwards, the work flow developed to perform the change detection is specified (Figure 3 ) considering both supervised and unsupervised approaches. Finally, remarks are provided discussing the benefits and limitations of the proposed methods.

\subsection{Data set}

The data set of images is obtained by a multi-temporal acquisition done in different époques (in 2006, in 2012 and in 2017).

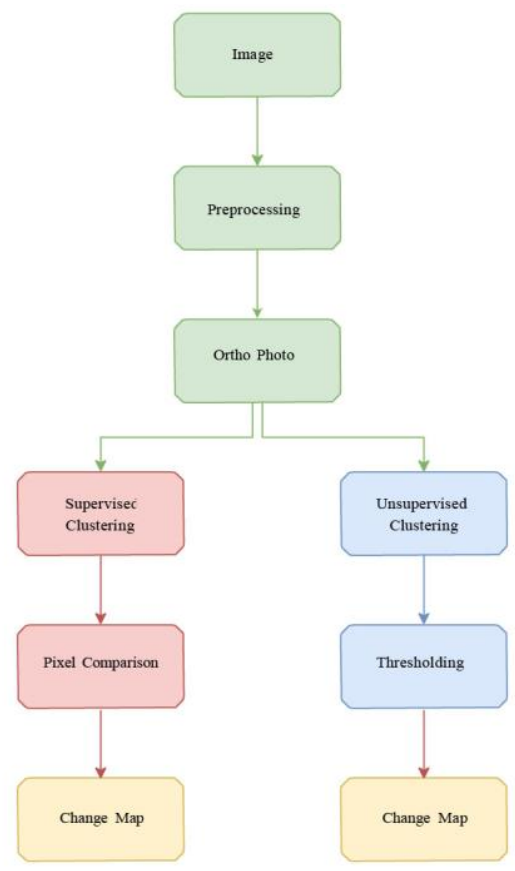

Figure 3. Workflow diagram for change detection

Images were collected for the purpose of achieving orthoimages, thus adopting a standard and well known procedure for close range photogrammetry (Furukawa et al., 2015). Albeit the acquisition was rigorous, the three image sets are not homogeneous, since they were obtained with different cameras; this implies different characteristics in terms of radiometric and geometric features, then fixed with image enhancement techniques. In 2006 the images of $3648 \times 2736$ were shot by a Panasonic Lumix DMC FZ50, focal length 7.4mm, 10.1 MP high resolution CCD. In the 2012 the set of images comes from a Canon Power shot SX30IS digital camera with focal length of $6.614 \mathrm{~mm}$, providing a maximum resolution of about 14.1 megapixel and images of $4320 \times 3240$. The 2017 data set is realized with SONY SLT-A77V camera with a focal length of $50 \mathrm{~mm}$ and a maximum resolution of 24 megapixel with images of $6000 \times 4000$ pixels. These different conditions require a preprocessing stage for noise removal and for the creation of three DEM and the relative ortho-images with comparable characteristic (GSD $2.428 \mathrm{~mm}$ ) to perform the change detection analysis, this includes geometrical rectification and image registration. To acquaint the reader about the subject of the experiment in the three époques (2006, 2012, 2017), an example is given in Figure 2, that was later cut (with blue dashed line) to keep the center of the bas relieves where the analysis, explained in $\operatorname{Sec} 4.2 .1$ and $\operatorname{Sec}$ 4.2.2.

\subsection{Change detection}

As stated at the beginning of this section, we followed a specific work flow (Figure 3). In particular, the process used in this paper starts from ortho-images where de-noising algorithm, applied only for the unsupervised approach, and other preprocessing operation were done; on these images, both supervised and unsupervised approaches were applied. At the end of the clustering, the process proceeds in parallel. On one side, for supervised clustering the change map is obtained with a pixel comparison is done. On the unsupervised clustering instead, the work goes on with a thresholding application to rise the change map.

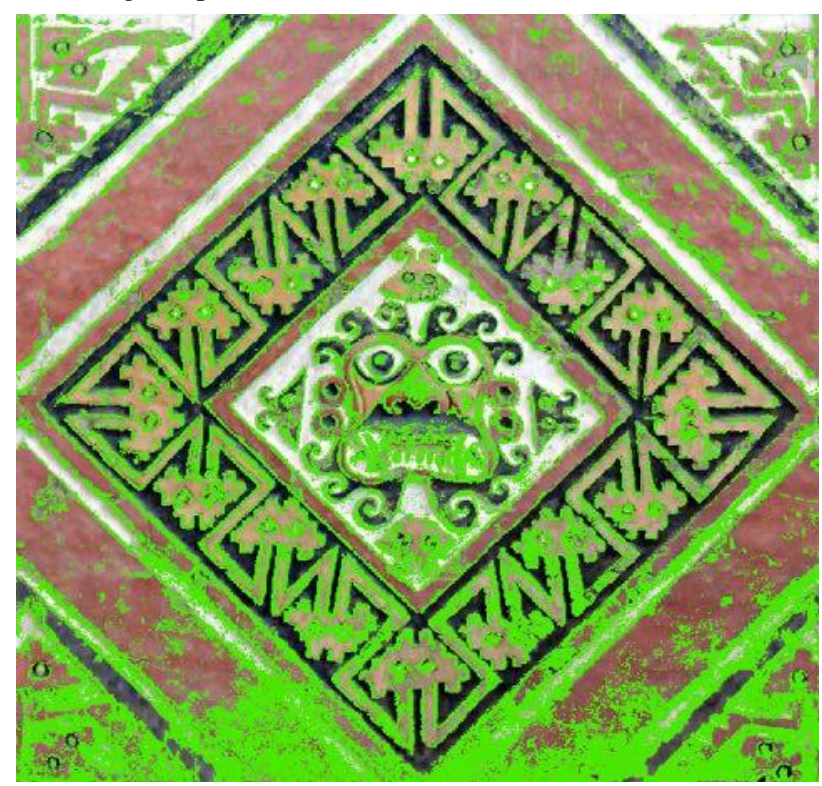

Figure 4. Result of per pixel subtraction between the data set of 2006-2017.

4.2.1 Supervised approach: This stage of the work follows our previous research (Malinverni et al., 2017), where a supervised approach was applied. The hard thematic map is used for comparison with the un supervised change map. The workflow to obtain this map is composed of the following steps. The first step, after a pre-processing based on image alignment and resampling, consists in creating DEM and ortho-images; this step is performed in Agisoft Photoscan without using GCP but identifying the same points in the three set of images as check points for the accuracy validation. The second step is based on the supervised classification with the use of maximum likelihood, which measures and divide the images in five classes 
identified by the user with a color based criteria; in particular, 4 classes are used for the principal pigments of the bass relieves and the last class is used to agglomerate the uncolored pixel in the images of the bass relieves. This classes are defined by an operator that creates a training and test set manually. After the classification, a pixel based subtraction is done and an example of the result is showed in the Fig. 4. The classification result is used as input of the segmentation algorithm, based on the Winner Takes All, that assigns the class to the right region and define the stability degree of that region. After this is possible to do a region based differential map that bring to the hard thematic map in Fig. 5.

4.2.2 Unsupervised approach: The unsupervised method, based on the work flow in Fig. 6 brings to the change map in Fig. 9. The unsupervised process uses a couple of images; for this reason is applied on the images [2006,2012] (where the squarebrackets identifies the couple and the first term is the old image and the second one is the newest image), [2012,2017], [2006,2017].

The application of unsupervised techniques for change detection is divided into three main sequential steps:

- pre-processing, reducing the noise and applying geometric and radiometric correction and co-registration;

- difference image generation, generated with the subtraction or ratio operator;

- analysis of the difference image, changes are detected with the K-Means clustering algorithm with the use of two clusters.

The process starts from two images, the old images (2006 and 2012 dataset) and the latest images (2017) of the bass relieves.

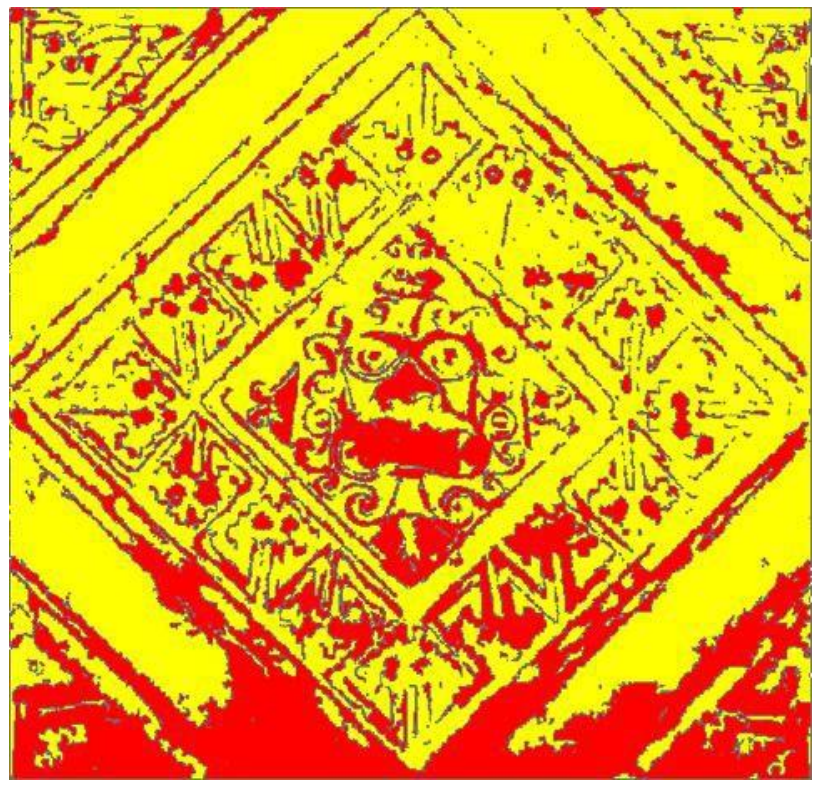

Figure 5. The supervised hard thematic map between 2006-

2017 after the winner takes all segmentation.

On this image, a pre-processing operation were done, in particular a de-noising operation. Then, we generated two different types of difference images: the first one based on the linear subtraction of the images obtained previously, the second one which applies a logarithmic subtraction on the same images. Finally, these difference images are combined with a linear function. The last step takes the combined difference image and applies the K-Means clustering algorithm with two clusters. The result of clustering was an image from where the thresholding application return the change map.

\section{Pre-processing}

Considering the two starting images the denoising function chosen is Non-Local means denoising algorithm (Buades et al., 2011). This algorithm replaces the color of a pixel with an average of the color of similar pixel, after scanning of vast portion of the image to find this similar pixel.

\section{Difference image generation}

This approach uses two different types of difference image. The first one is based on the subtraction of the two starting images and the second, instead of the classic ratio operator, uses a logarithmic approach to find the image results of division. The two images generated by the two differences operator are filtered, the subtraction image is filtered by a mean filter, because there are several isolated pixels and with this filter the image becomes more consistent. The log image is filtered with a median filter to preserve the edge information. At the end of this step is created a linear combination image of the two previous images with a linear combination. Thanks to the filter stage this combined image has a strong local consistency and preserves the shape of the changed area. An example of the result of combined difference images is in Fig.7.

\section{Change detection}

The last step consists in using K-means and thresholding to obtain a change map. In particular, $\mathrm{K}$-means is used to partition the combined difference image into two cluster: changed and unchanged. The resulting image is represented in Fig. 8. With theresholding of the clusterization a change map is obtained. This operation is done using the Otsu thresholding algorithm, that is showed in (Goh et al., 2018), is the best thresholding algorithm in image processing, since an arbitrary thresholding

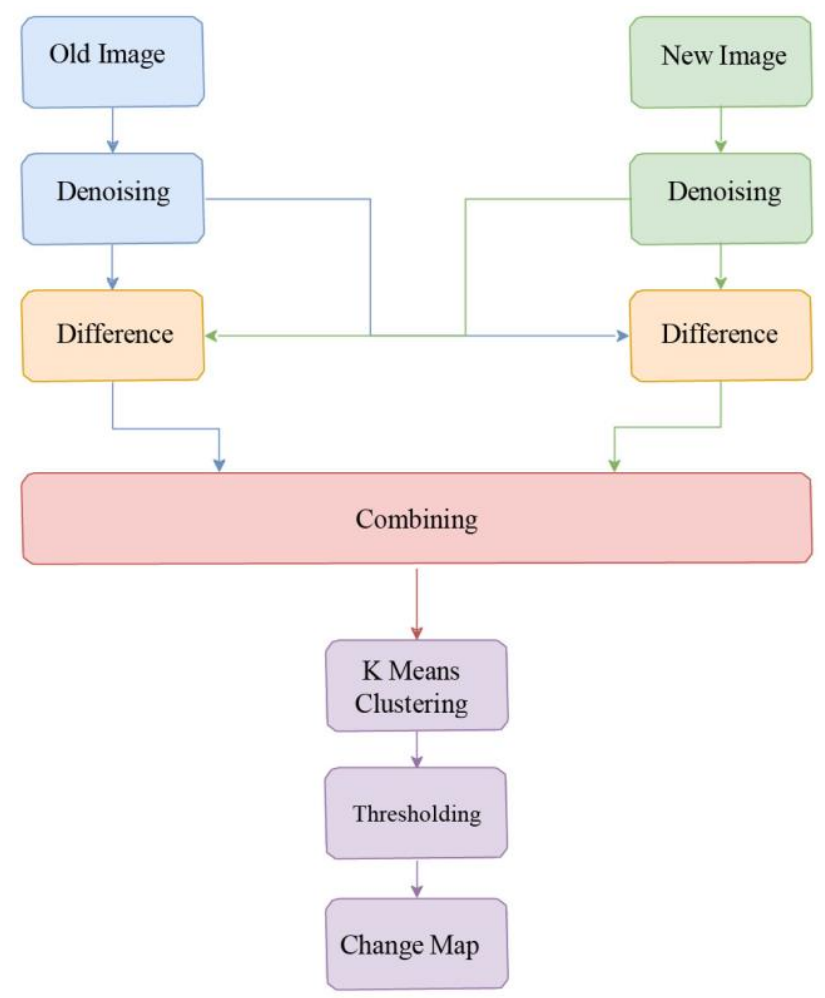

Figure 6. Workflow diagram for unsupervised change detection 
approach. The change-map obtained is a binary image as showed in Fig.9, that was then elaborated to visualize the effective changes over the bass relieve, as showed in Figure 10.

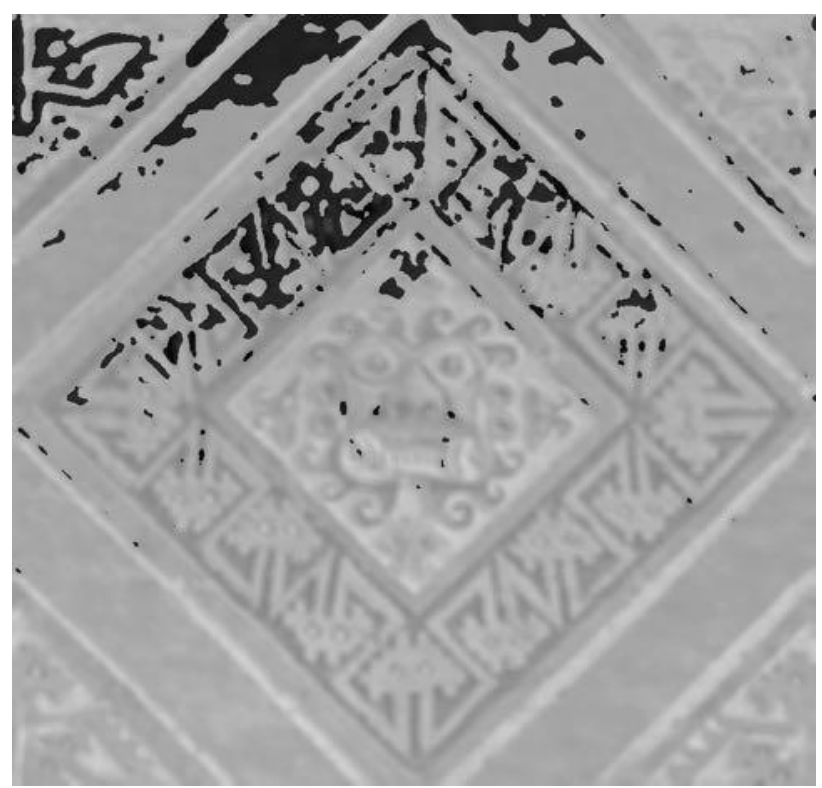

Figure 7. Combined difference image of 2006 - 2017 outperforms

\section{RESULTS}

This section analyzes and compares the results obtained from the two methods described in Sec 4. At the end concludes that the supervised clustering outperforms the unsupervised approach. The comparison between the changes in 2012 and 2017 from 2006 in term of pixels shows that the $3 \%$ of pixels is changed. In particular, 19588 pixels are changed in the dataset obtained in years 2012 and 2017 compared with the dataset acquired in the 2006 . In the 2012 , there are $0.37 \%$ pixel (i.e., 2389 pixels) not resulting an effective change in 2017. Furthermore, in the 2017 there $4.55 \%$ (i.e., 29652 pixels) of pixel changed that in the 2012 was not changed. For the sake of completeness, results are reported in Table 1.

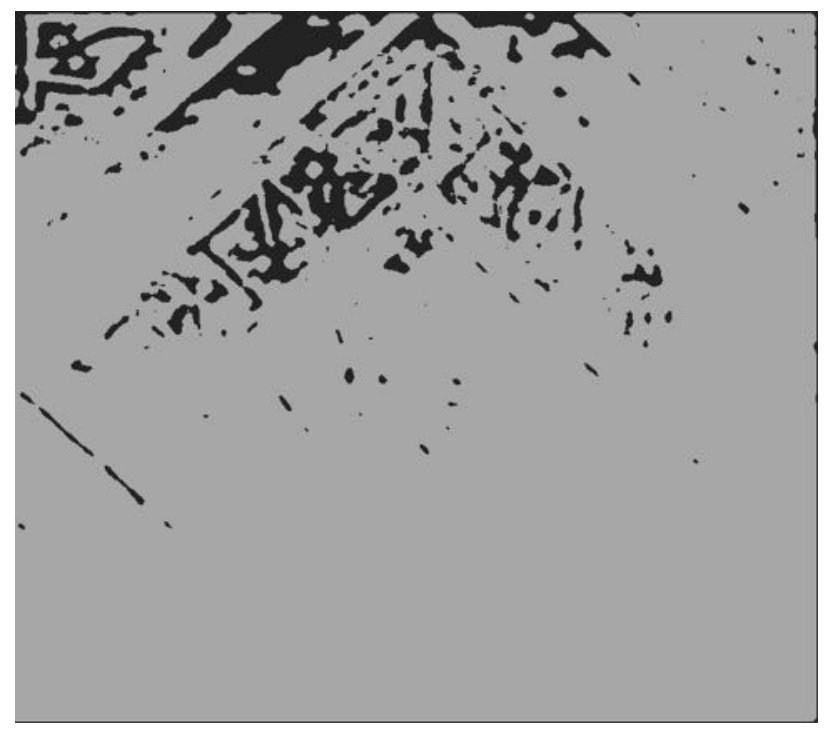

Figure 8. The clustered combined difference image between 2006-2017

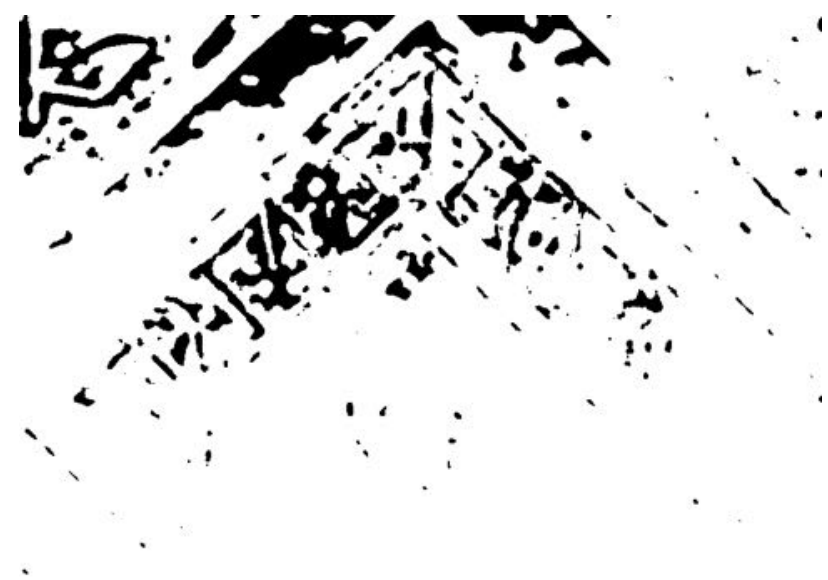

Figure 9. The unsupervised change-map between 2006-2017, The difference images is computed in such a way that the resulting image has the pixel where changes are effective with a high gray level instead of the pixel where the changes are irrelevant or absent with a low gray level.

\begin{tabular}{c|cccccc} 
Change & 1-red & 2-white & 3-yellow & 4-blue & 5-gray & total \\
\hline 2012 & 642 & 304 & 277 & 96 & 1070 & 2389 \\
2017 & 1874 & 348 & 12191 & 10659 & 4580 & 29652 \\
$2012-2017$ & 16056 & 21 & 1006 & 1145 & 1360 & 19588
\end{tabular}

Table 1. Number of pixels changed for class and year

From the computation, we obtained Fig. 9 that shows the results of the supervised clustering from (Malinverni et al., 2017) on the 2017 data set with respect to the 2006. As visible, the results show a homogeneity on changes that is more realistic. The Table 3 shows the changes between the images of 2006 and 2017.

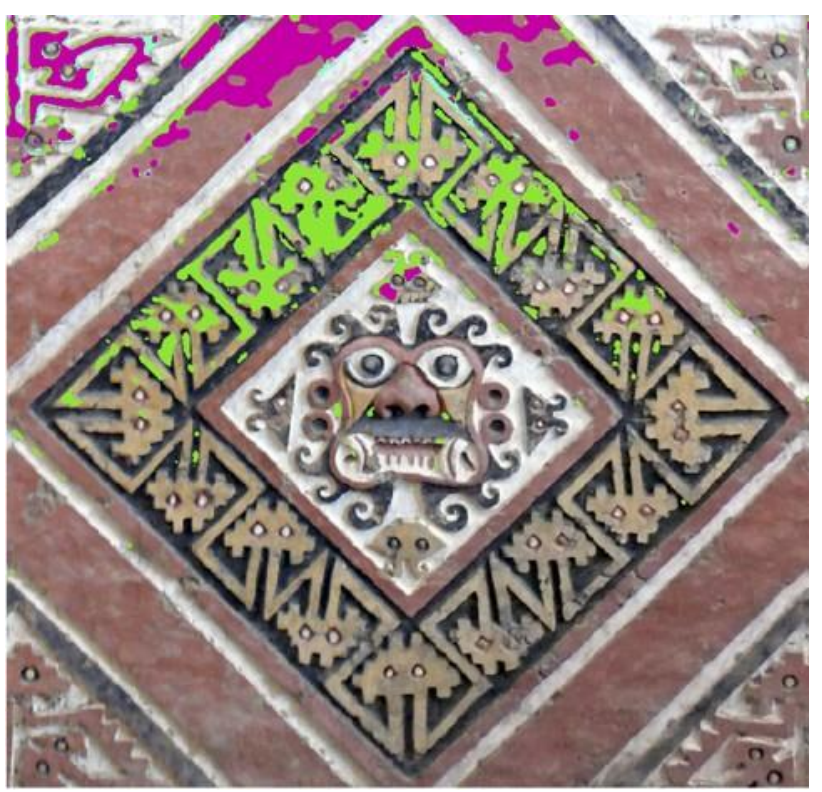

Figure 10. The Image where there are all the changes over the year. Legend: cyan is for the changes detected only in 2012; green is for the changes detected only in 2017; magenta is for the changes that are in both 2012 and 2017 


\begin{tabular}{c|ccccc|c} 
Ground Truth / Predicted Class & 1-RED & 2-WHITE & 3-YELLOW & 4-BLUE & 5-GREY & Class-Specific Recall \\
\hline 1-RED & 3522 & 30 & 134 & 286 & 2 & $88,63 \%$ \\
2-WHITE & 2 & 3644 & 441 & 3 & 237 & $84,22 \%$ \\
3-YELLOW & 5 & 30 & 3777 & 12 & 26 & $98,10 \%$ \\
4-BLUE & 0 & 0 & 0 & 2973 & 17 & $99,43 \%$ \\
5-GRAY & 3 & 144 & 1 & 68 & 1073 & $83,24 \%$ \\
\hline Class-Specific Precision & $99,72 \%$ & $94,70 \%$ & $86,77 \%$ & $88,96 \%$ & 79, & $91,23 \%$
\end{tabular}

Table 2. Confusion Matrix of supervised clustering on the 2017 data set

\begin{tabular}{|c|c|c|c|c|c|c|c|}
\hline 2006 vs 2017 (pixel) & $1-\mathrm{red}$ & 2 - white & 3 - yellow & 4 - blue & 5 - gray & Total per class (2006) & Changed from $2006(\%)$ \\
\hline $1-$ red & 167049 & 1248 & 16647 & 10198 & 12384 & 207526 & 19 \\
\hline 2 - white & 1424 & 78561 & 9607 & 2901 & 14368 & 106.861 & $50 \%$ \\
\hline 3 - yellow & 4132 & 5872 & 60210 & 8754 & 9928 & 88896 & 26 \\
\hline 4 - blue & 1919 & 2031 & 7077 & 110974 & 11828 & 133829 & $48 \%$ \\
\hline 5 - gray & 11872 & 14983 & 21568 & 34340 & 33488 & 116251 & 32 \\
\hline Total per class (2017) & 186396 & 102695 & 115109 & 167167 & 81996 & 653363 & 31 , \\
\hline
\end{tabular}

Table 3. Region based comparison between 2006 and 2017 acquisitions

\begin{tabular}{c|ccccc|c|c}
2006 vs $2017\left(\mathrm{~mm}^{2}\right)$ & $1-$ red & 2 - white & 3 - yellow & $4-$ blue & 5 - gray & Total per class $(2006)$ & Changed from 2006 $(\mathbf{\%})$ \\
\hline 1 - red & 1062083 & 8397 & 67485 & 68113 & 43830 & 1249908 & 15, \\
2- white & 12695 & 456172 & 31364 & 21377 & 77864 & 599472 & $03 \%$ \\
3 - yellow & 6863 & 31196 & 425138 & 15324 & 30019 & 508540 & 23, \\
4 - blue & 8803 & 6323 & 16435 & 715973 & 21597 & 769131 & $90 \%$ \\
5 - gray & 67534 & 67958 & 142797 & 174542 & 203736 & 656567 & 16, \\
\hline Total per class (2017) & 1157978 & 570046 & 683219 & 995329 & 377046 & 3783618 & 24,
\end{tabular}

Table 4. Comparison between the data set of 2006 and 2017 with the expansion of the area change in $\mathrm{mm}^{2}$

The mean change is $31,08 \%$ and the most significance change in percentage is in the yellow class with $32,27 \%$. The Table 4 reports the amount of the area changed from 2006 data set in the 2017 data set and is immediately visible that the highest area changes are for gray color. The Table 2 confirms that the supervised methods is better than the unsupervised approach, indeed the confusion matrix exhibits the precision and the recall based on the preliminary knowledge of the bass relieves and the results on this measure shows the reliability of this approach with respect to the K-Means unsupervised approach. The comparison with the changes obtained from the work flow explained in Sec 4.2.2 brings to the conclusion that the unsupervised techniques is not the right way to proceed for the change detection in the $\mathrm{CH}$ domain, although the supervised algorithm, with the human interaction, is the better approach instead of the unsupervised one.

\section{CONCLUSION}

Following a previous study, this work describes a methodology to uncover color changes over ancient bass relieves. With respect to the previous study, the discussion was widen describing the restoration project that is carried out the local community, on the Huaca de la Luna. Beside this, a new dataset collected in 2017 was included in the flow of change detection, in order to monitor the state of color decay of the artifact. To complete the validation of the method, unsupervised and unsupervised methods have been used on the same set of images, in order to under- stand which is the best approach to adopt in such scenario. The results of the comparison, described in Section 4., are both qualitative and quantitative. It can be observed that in the unsupervised change map, changes are concentrated only on the upper part of the of the image; conversely, the supervised one changes are more distributed and homogeneous. This is even confirmed by the Tables 4 and 3 which reports the values of change for each class. The supervised methods outperform the unsupervised one; the latter in fact cannot be considered reliable for the purpose of this study. We can thus conclude that the pipeline of work already developed for detecting color changes over time is reliable. What remains open for the future, that we foresee to face in the upcoming months, is to validate the method directly in the field even with expert archaeologist. This means collecting a real ground truth that might help to ascertain the output of the classification, hence of the stability map. Another important aspect to whom we will dedicate our efforts will be to develop a GIS based monitoring system in which the spatio temporal information could be collected and managed for the bass relieves subject o this study and for many other that are present in the archaeological site.

\section{REFERENCES}

Alba, M. and Scaioni, M., 2010. Automatic detection of changes and deformation in rock faces by terrestrial laser scanning. Int. Arch. Photogramm., Remote Sens. Spatial Inf. Sci., $38,11-16$.

Appolonia, L. et al., 2006. Computer-aided monitoring of buildings of historical importance based on color. Journal of Cultural Heritage 7.2, 85-91.

Atkinson, A.D.J., de Sanjosé Blasco, J.J. and de Matias Bejarano, J., 2010. Applied 3D Photogrammetric Studies for the Historical Heritage of Extremadura (Spain). ISPRS Commission V, ISSN, 1682-1777.

Barazzetti, L. et al., 2010. Geometric and radiometric analysis of paintings. Int. Arch. Photogramm., Remote Sens. Spatial Inf. Sci.,38. Part 5. 
Bourget, S. and L. Jones, K., 2009. The art and archaeology of the Moche: an ancient Andean society of the Peruvian North Coast. University of Texas Press.

Buades, A., Coll, B. and Morel, J.M., 2011. Non-local means denoising. Image Processing OnLine 1, 208-212.

Burroni, D. et al., 2002. The surface alteration features of flint artefacts as a record of environmental processes. Journal of Archaeological Science 29.11, 1277- 1287.

Cerra, D. et al., 2016. Automatic damage detection for sensitive cultural heritage sites. International Society for Photogrammetry and Remote Sensing.

Furukawa, Y., Hernandez, C. et al., 2015. Multi-view stereo: A tutorial. Foundations and Trends in Computer Graphics and Vision 9.1-2, 1-148.

Gamarra, M., 2003. Iconografía litúrgica y contexto arquitectónico en Huaca de la Luna, valle de Moche. Moche: Hacia el final del milenio, edited by Santiago Uceda and Elias Mujica, 425-476.

Yang Goh, T. et al., 2018. Performance analysis of image thresholding: Otsu technique. Measurement 114, 298-307.

Levin, S. and Filin, S., 2010. Archaeological site documentation and monitoring of changes using surface-based photogrammetry. ISPRS Commission V Mid-Term Symposium Close Range Image Measurement Techniques, 21-24.

Liciotti, D. et al., 2018. Convolutional networks for semantic heads segmentation using top-view depth data in crowded environment. 2018 24th International Conference on Pattern Recognition (ICPR), IEEE, 1384-1389.

Lu, D. et al.,2004. Change detection techniques. International journal of remote sensing 25.12, 2365-2401.

Mahulkar, H.N. and Sonawane, B., 2016. Unsupervised approach for change map generation. 2016 International Conference on Communication and Signal Processing (ICCSP), IEEE, 0037-0041.

Malinverni, E.S. et al., 2017. Documentation and detection ofcolour changes of bas-relieves using close range photogrammetry. Int. Arch. Photogramm., Remote Sens. Spatial Inf. Sci., 42.

Morales, R., Solórzano, J. and Asmat, M., 1996. Superficies arqui-tectónicas: tipología, tecnología y materiales. Investigaciones en la Huaca de la Luna, 211-219.

Paolanti, M. et al., 2018. Person Re-Identification with RGB- D Camera in Top-View Configuration through Multiple Nearest Neighbor Classifiers and Neighborhood Component Features Selection. Sensors 18.10, 3471.

Pierdicca, R. et al., 2018. Deep Convolutional Neural Network for Automatic detection of damaged photovoltaic cells. Int. Arch. Photogramm., Remote Sens. Spatial Inf. Sci., 42.2.
Uceda, S. and Morales, R., 2010. Moche, pasado y presente, Trujillo, Patronato Huacas del Valle de Moche, Fondo Contravalor Perù-Francia y Universidad Nacional de Trujillo.

Shao, P. et al., 2016. Novel approach to unsupervised change detection based on a robust semi-supervised FCM clustering algorithm. Remote Sensing 8.3, 264.

Uceda, S., 2001. Investigations at Huaca de la Luna, Moche Valley: An Example of Moche Religious Architecture. Studies in the History of Arts.

Uceda, S., Morales, R. and Mujica, E., 2016. Huaca de la Luna: templos y Dioses Moches. WM, World Monuments Fund Perù.

Vergès-Belmin, V., 2008. ICOMOS-ISCS: Illustrated glossary on stone deterioration patterns, Monuments and sites $X V$, Paris, ICOMOS.

Wright, V., 2004. Analyses préliminaires de caractérisation de supports et de pigments de reliefs polychromes, Huacade la Luna, Trujillo, Pérou. British Archaeological Reports (BAR S1389), Paris Monographs in American Archaeology 16, Archaeopress, Oxford, England.

Wright, V., 2010. Pigmentos y tecnología artística mochicas: una nueva aproximación en la comprensión de la organización social. Bulletin de l'Institut franc, ais d'e'tudes andines 39 (2), 299-330.

Yuan, Y., Lv, H. and Lu, X., 2015. Semi-supervised change detection method for multi-temporal hyperspectral images. Neurocomputing 148, 363-375. 